\title{
Percutaneous balloon compression for the treatment of trigeminal neuralgia: results in 56 patients based on balloon compression pressure monitoring
}

\author{
Jeffrey A. Brown, M.D., and Julie G. Pilitsis, M.D., Ph.D. \\ Department of Neurological Surgery, Wayne State University School of Medicine, Detroit, Michigan
}

\begin{abstract}
Object. Percutaneous balloon compression is an effective and technically simple method for treating trigeminal neuralgia (TN). Nevertheless, dysesthesias (10-20\%) and masseter muscle weakness (66\%) following the procedure have been noted. The purpose of this study was to evaluate the results of testing TN with percutaneousballoon compression aided by intraluminal pressure monitoring.

Methods. In this study the authors review the results and complications associated with percutaneous balloon compression by using intraluminal pressure monitoring data obtained in 65 procedures performed in 56 consecutive patients over 4 years. The mean patient age was 71 years (range 37-92 years), and the mean follow-up duration was 17 months (range 3-38 months). The mean intraluminal compression pressure was (1160 $\pm 62 \mathrm{~mm} \mathrm{Hg}$ ), and the mean duration of compression was $1.15 \pm 0.27$ minutes. The trigeminal depressor response was observed in 60 (92\%) of 65 procedures, and initial pain relief occurred in $92 \%$ of patients. The recurrence rate in patients who had initial relief was $16 \%$ (nine of 56). The mean time until recurrence in patients who experienced pain relief after surgery was 13 months (range 3-23 months). Mild numbness immediately after surgery was observed in $83 \%$ of patients. At the most recent evaluation, $17 \%$ of patients reported persistent, nontroublesome numbness and none had moderate or severe numbness. Minor dysesthesia was present in two patients (4\%). Mild masseter muscle weakness occurred in $24 \%$ of patients and resolved within a maximum period of 1 year. No patient experienced anesthesia dolorosa, corneal keratitis, or other cranial nerve deficits. These morbidity rates are lower than the incidence reported in the literature when pressure monitoring is not used.

Conclusions. These data show that by monitoring compression pressure and limiting the duration of compression, it is possible to reduce the incidence of dysesthesias, severe numbness, and masseter weakness after surgery without increasing the rate of recurrent pain in patients with classic TN.
\end{abstract}

KEY WORDS - trigeminal neuralgia • trigeminal nerve • pain • compression

In the 1950s, neurosurgeons frequently performed intraoperative manipulation of the trigeminal ganglion and the maxillary and mandibular divisions to treat the pain of TN. ${ }^{25}$ Based on this concept, Sean Mullan introduced the technique of percutaneous balloon compression for TN in 1983..$^{21,22}$ Since that first publication, results from more than 800 of these procedures have been reported in the lit-

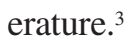

Balloon compression has the advantage of selectively avoiding injury to the small, unmyelinated fibers that mediate the corneal reflex, providing relative protection to corneal sensation in first-division TN. ${ }^{6}$ Balloon compression rarely causes anesthesia dolorosa or corneal keratitis and can be performed with the patient in a state of general

Abbreviations used in this paper: MR = magnetic resonance; $\mathrm{TN}=$ trigeminal neuralgia. anesthesia, limiting the pain and anxiety that are inherent in other percutaneous procedures. ${ }^{20}$ Nevertheless, previous studies have demonstrated a higher incidence of masseter muscle weakness and dysesthesias after balloon compression than with other ablative procedures. ${ }^{8,17}$ The incidence of mild masseter weakness with balloon compression is reportedly as high as $100 \%,{ }^{17}$ whereas the reported incidence of dysesthesias ranges from 10 to $20 \% .^{7,17}$ To avoid these complications, we sought to reduce the injury to the trigeminal nerve that is needed to treat patients with TN successfully. These efforts to lessen compression of the trigeminal nerve require a shorter compression time and that the intraluminal balloon pressure be monitored continuously. The purpose of this retrospective study was to examine the results of balloon compression in a consecutive series of patients who underwent the procedure combined with intraluminal pressure monitoring during surgery. 


\section{CLINICAL MATERIAL AND METHODS}

\section{Patient Population}

Sixty-five percutaneous balloon compressions were performed in 56 consecutive patients with classic symptoms of unilateral TN between 2000 and 2004. As shown in Table 1, the mean age of the patients was 71 years (range 37-92 years). The mean follow-up duration was 17 months (range 3-38 months). Twenty-three patients (with $36 \%$ of the procedures) had undergone previous destructive procedures before their balloon compression. In 10 of 56 patients (with $15 \%$ of the procedures) there was first-division pain. Six patients $(11 \%)$ had multiple sclerosis.

All patients had been treated with carbamazepine or gabapentin for trigeminal pain before being considered for surgery. Preoperative MR imaging with Gd enhancement was routinely performed. When MR imaging was not possible, computerized tomography scans were obtained, with $5-\mathrm{mm}$ slices in the region of the Meckel cave. None of the patients had an associated tumor. The indications for surgery were similar to those for other percutaneous procedures for treatment of TN. Balloon compression was chosen for patients in the following groups: 1) elderly or infirm patients who were at higher risk for morbidity from microvascular decompression; 2) young patients who sought a percutaneous operation with an expectation of minimal postoperative numbness; and 3) those in whom medical therapy failed or who could not tolerate medical therapy with anticonvulsant medications. Preoperative and postoperative data were obtained from review of the medical charts and postoperative questionnaires. Data analysis was performed using commercially available software (SPSS, Inc., Chicago, IL), and a probability value of less than 0.05 was considered statistically significant. The Institutional Review Board at Wayne State University approved this study.

\section{Surgical Technique}

Surgery was performed in the operating room, not the radiology suite, with the aid of fluoroscopic imaging. A light

TABLE 1

Characteristics of 56 patients who underwent percutaneous balloon compression for $T N^{*}$

\begin{tabular}{lc}
\hline \hline \multicolumn{1}{c}{ Characteristic } & Value (\%) \\
\hline age (yrs) & $71.4 \pm 10.5$ \\
sex $(\mathrm{M} / \mathrm{F})$ & $23: 33$ \\
disease duration (yrs) & $7.5 \pm 4.6$ \\
dist most affected/no. of ops & $10 / 65(15.4)$ \\
$\mathrm{V}_{1}$ & $25 / 65(38.5)$ \\
$\mathrm{V}_{2}$ & $30 / 65(46)$ \\
$\mathrm{V}_{3}$ & 38 \\
prior op $(\%)$ & 8 \\
none & 54 \\
$\mathrm{MVD}$ & $17.5 \pm 7.8$ \\
destructive lesioning & 16 \\
time since op (mos) & $12.6 \pm 4.6$ \\
rate of recurrence $(\%)$ & \\
time to recurrence (mos) &
\end{tabular}

\footnotetext{
* Results are expressed as the mean \pm standard error of the mean.
} Abbreviations: dist = distribution; MVD = microvascular decompression. general anesthesia was induced, and then an external pacemaker was placed on the patient's chest. The pacemaker was set to trigger if the heart rate fell below 45 beats/minute and was carefully tested prior to draping. This trigeminal depressor response seen during trigeminal nerve compression consists of both bradycardia and brief hypotension, often with a reflex hypertension emerging after the pacemaker is triggered. Preoperative atropine was not given so that we could use the depressor response as a monitor of trigeminal compression.

Patients were positioned supine, with a roll under their shoulders, allowing approximately $15^{\circ}$ of extension. A Food and Drug Administration-approved kit that included an introducing cannula, sharp and blunt obturators, curved and straight guiding stylets, and a No. 4 balloon was used to perform the surgery (Cook Vascular, Inc., Leechburg, PA). The 14-gauge introducing cannula was percutaneously passed to the foramen by using Härtel guidelines and aided by lateral fluoroscopic imaging. For third-division pain, the cannula was directed toward the foramen ovale, nearly parallel to the slope of the petrous bone as seen on the lateral image.

A submental view was obtained once the cannula reached the skull base. When the foramen ovale was identified on the fluoroscopic image, the cannula was advanced under repeated, intermittent fluoroscopic guidance by using the submental view. This view provides direct visualization of the foramen ovale. It is easy to feel when the 14-gauge cannula engages the foramen ovale. Often a brief trigeminal depressor response occurs from mechanical compression of the mandibular nerve at the foramen. The cannula should not pass beyond the foramen ovale, and cerebrospinal fluid is not seen when the cannula is properly positioned.

Once the cannula had engaged the foramen ovale, the blunt obturator was removed and a straight guiding stylet inserted. Using the anteroposterior image (with the petrous ridge positioned in the radiographic center of the orbit) the stylet was directed at the medial dip in the petrous bone, which is the proximal entrance to the Meckel cave, the porus trigeminus. The stylet was pointed toward the center of the porus for second- or multidivision pain; to the lateral porus for third-division pain; and to the medial porus for first-division pain. The entrance to the porus is found approximately $17 \mathrm{~mm}$ beyond the foramen ovale. The tip of the stylet was set approximately $2 \mathrm{~mm}$ beyond the edge of the petrous ridge as seen through the orbit. Once properly positioned, the stylet was withdrawn and the balloon catheter inserted into the same site.

The lateral view of the skull was used during the balloon compression. For second- or third-division pain, the stylet remained parallel and adjacent to the petrous bone. For second- and first-division pain, the stylet was positioned at a more oblique angle relative to the plane of the petrous bone. Once the proper trajectory was established, the stylet was removed. Air was evacuated from the balloon with a tuberculin syringe connected to a three-way stopcock. An insufflation syringe (Merit Medical, Inc., Salt Lake City, UT) was connected to the balloon and used to measure intraluminal pressures in atmospheres of pressure. The balloon was inflated with 0.7 to $0.75 \mathrm{ml}$ of $180 \mathrm{mg} \%$ iohexol to reach a target intraluminal compression pressure of 1065 
to $1215 \mathrm{~mm} \mathrm{Hg}$. Once that pressure was reached and a corresponding pear shape observed, the balloon was left inflated for 1 to 1.5 minutes. The pear shape indicates that the tip of the balloon lies within the porus trigeminus, which is where it must lie to compress the trigeminal nerve adequately. The depressor response may occur at this point, briefly triggering the external pacemaker. Intermittent lateral imaging was obtained to monitor the balloon's position and shape. The needle and catheter were then removed, and an ice pack and sterile bandage applied to the cheek. The patient was discharged on the day of surgery or the following morning.

\section{RESULTS}

\section{Clinical Data}

Initial pain relief was attained in $92 \%$ of patients. Six patients had minimal or no initial pain relief; four of them had evidence of vascular compression on MR imaging, but had elected to undergo balloon compression as their initial therapy. These patients subsequently underwent microvascular decompression and attained pain relief. The remaining two patients had both a classic electric-shock and a burning, neuropathic component to their pain. This may explain the difficulty in treating them, because there was an element of more atypical pain rather than classic TN.

There were nine recurrences (14\%) among the patients who had satisfactory pain relief initially. Subsequent balloon compressions were performed in eight of these nine patients, and pain relief was obtained in all eight. Among the 56 patients, $35 \%$ had undergone previous destructive peripheral procedures. This higher incidence of patients who had previously undergone surgery may have led to a higher recurrence rate in this series. Kaplan-Meier survival curves were calculated and the mean time until recurrence was 13 months (Fig. 1).

Immediately after surgery, $83 \%$ of patients reported mild numbness. At the most recent postoperative visit, $17 \%$ of patients reported persistent numbness. No patient described this numbness as being moderate or severe. Two patients (4\%) reported minor dysesthesias. Mild masseter muscle weakness was reported in $24 \%$ of patients, and it resolved

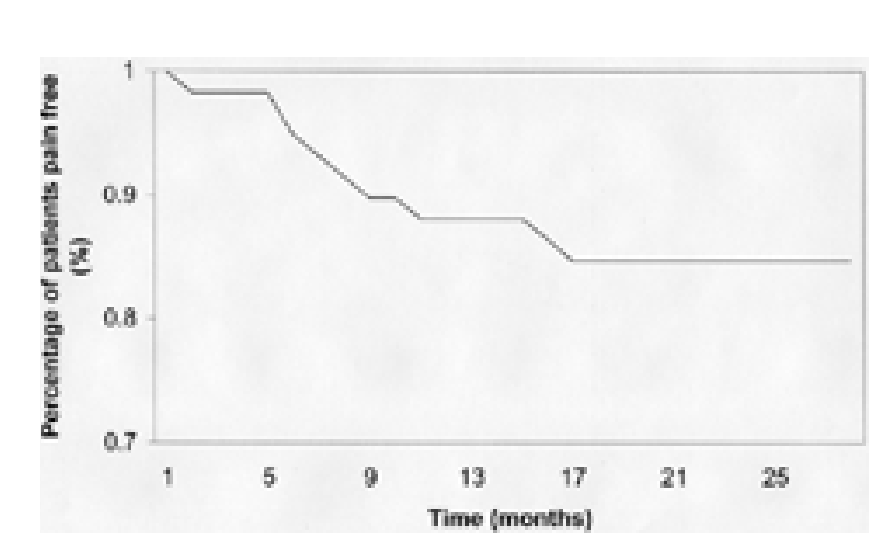

Fig. 1. Kaplan-Meier analysis of pain recurrence following balloon compression for TN in patients who experienced initial pain relief. The mean time until recurrence was 13 months. Results are expressed as the mean values \pm standard error of the mean. in all of them in a maximum of 1 year. Neither anesthesia dolorosa nor corneal anesthesia occurred. No other cranial nerve deficits due to compression occurred. No patient suffered aseptic meningitis. There was no significant correlation between the duration of preoperative pain and the likelihood that masseter muscle weakness or sensory dysesthesias would develop.

One death occurred: this patient had undergone previous microvascular decompression, and when pain recurred he underwent balloon compression. He suffered diffuse subarachnoid hemorrhage from a dural arteriovenous fistula found on postoperative angiography studies. This fistula was not evident on the MR image obtained before surgery.

\section{Intraoperative Variables}

The mean intraluminal balloon compression pressure was $1160 \pm 62 \mathrm{~mm} \mathrm{Hg}$. The mean duration of compression was $1.15 \pm 0.27$ minutes. The depressor response was observed in 60 of 65 procedures. Intraluminal pressure did not significantly affect the development of numbness or masseter muscle weakness (Table 2). There was a nonsignificant trend for patients whose intraluminal pressure was maintained at 1140 to $1216 \mathrm{~mm} \mathrm{Hg}(1.5-1.6 \mathrm{~atm})$ to have a lower incidence of failure or recurrence (chi-square test -3.81 , df $1, p=0.1 ;$ Fig. 2). The presence or absence of the depressor response also did not affect morbidity or recurrence. The duration of compression significantly affected the development of postoperative numbness $(\mathrm{p}<0.03)$.

\section{DISCUSSION}

Percutaneous balloon compression is a simple and effective treatment for TN that has been used for more than two decades. The rate of pain relief after balloon compression is similar to that reported for radiofrequency thermocoagulation and higher than that associated with glycerol radiolysis or stereotactic radiosurgery. ${ }^{17}$ Balloon compression is especially useful in patients with first-division pain because it does not injure the myelinated fibers that mediate the blink reflex and thus does not lead to corneal keratitis. Masseter muscle weakness, severe numbness, and dysesthesias have been noted, however. Our goal in this series of patients was to produce a trigeminal nerve injury that was

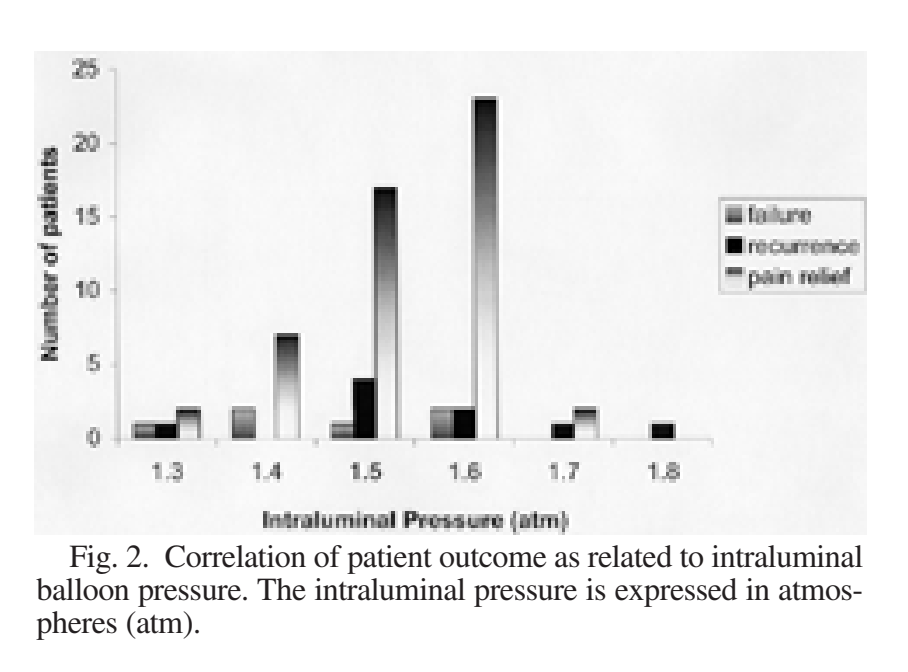


TABLE 2

Effect of intraoperative variables on patient outcome after percutaneous balloon compression for $T N^{*}$

\begin{tabular}{lcc}
\hline \multicolumn{1}{c}{ Variable } & Pressure $(\mathrm{mm} \mathrm{Hg})$ & Time $(\mathrm{mins})$ \\
\hline numbness & & \\
present & $1550 \pm 75$ & $1.18 \pm 0.23$ \\
absent & $1520 \pm 81$ & $1.06 \pm 0.10$ \\
p value & 0.15 & 0.03 \\
masseter weakness & $1536 \pm 69$ & $1.11 \pm 0.18$ \\
present & $1523 \pm 85$ & $1.17 \pm 0.21$ \\
absent & 0.32 & 0.23 \\
p value & $1556 \pm 106$ & $1.14 \pm 0.19$ \\
pain recurrence & $1528 \pm 75$ & $1.15 \pm 0.20$ \\
yes & 0.29 & 0.43 \\
no & & \\
p value &
\end{tabular}

* Results are expressed as the mean \pm standard error of the mean.

enough to relieve pain consistently, but that limited the occurrence of other complications. By monitoring the compression pressure and modifying its duration, in this study we achieved a rate of pain relief that was comparable to that reported in other studies of balloon compression for TN, but with a lower incidence of dysesthesias, masseter weakness, and severe numbness.

In previous studies of the results of balloon compression for TN, investigators have reported dysesthesias in 10 to $20 \%$ of patients and moderate to severe numbness in $20 \%$ following this procedure. ${ }^{4,7,15,17}$ Masseter muscle weakness has been recognized as common and its incidence is not defined. In a recent review of the literature, Lopez, et al., state that such weakness occurs in nearly $100 \%$ of patients. Transient additional cranial nerve deficits, presumably related to overcompression, have been reported in $1.5 \%$ of patients. $7,16,17$

In our study, dysesthesias occurred in $4 \%$ and mild masseter weakness in $24 \%$ of patients. No moderate or severe numbness was produced and there were no other cranial nerve deficits. Neither anesthesia dolorosa nor corneal keratitis occurred. Pain relief occurred in $92 \%$ of patients. At a mean follow-up duration of 17 months, the recurrence rate was $16 \%$, which is similar or even better than in our previous series. ${ }^{7}$ The first author (J.A.B.) performed all operations in both series.

The main difference between this series and previous studies by the first author is the consistent use of a pressuremonitoring device. ${ }^{89}$ It was used in a small subset of patients in the last report, but is now used in all cases. ${ }^{8}$ The target compression pressure was between 988 and $1368 \mathrm{~mm}$ $\mathrm{Hg}$. The resulting tissue compression pressure was estimated to be 750 to $1250 \mathrm{~mm} \mathrm{Hg}$. ${ }^{4}$ The duration of the compression is also monitored. In the previous series, compressions lasting up to 3 minutes were performed in recurrent cases. ${ }^{7}$ In this series, the mean compression time was 1.15 minutes. Our data showed that compression time directly correlated with the development of numbness. Although the pressure data did not reach statistical significance, there was a trend for a better outcome, that is, a lower recurrence rate, in patients whose intraluminal compression pressure measured between 1140 and $1216 \mathrm{~mm} \mathrm{Hg}$.

In the last two decades there has been wide variation in the amount and duration of compression pressure used during surgery. For example, pressure measurements between 980 and $2080 \mathrm{~mm} \mathrm{Hg}$ have been described. ${ }^{16}$ One group used a compression time of 3.5 minutes and found that a low pressure resulted in a higher recurrence rate $(75 \%)$ but fewer side effects $(0 \%)$, whereas with a high pressure there were no recurrences at the 1-year follow-up review, but $100 \%$ of the patients had side effects. ${ }^{29}$ Studies have also been conducted to find the most appropriate pressure. In one study performed in cadavers, researchers found that the mean intraluminal pressure of a Fogarty catheter balloon in the Meckel cave was $1266 \pm 80 \mathrm{~mm} \mathrm{Hg} .{ }^{9}$ In a recent study in 62 patients, investigators found that the mean compression pressure of the Fogarty catheter in the Meckel cave

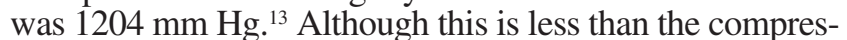
sion pressures used in our study, it is probably because the pressure dynamics of the balloon used in the Cook kit and the Fogarty balloon are not comparable. Compression times ranging from 1 to 7 minutes have been reported and sensory loss was subjectively noted to increase with the duration of compression. $^{12}$

Although many groups have documented compression pressure, few have consistently monitored amount of compression, duration of compression, and balloon volume. In a recent study, however, researchers held pressure and volume stable while they altered the total duration of balloon compression. The patients who received compression for 3 minutes had significantly more sensory complaints than the group of patients who had 1 minute of compression. ${ }^{14}$ In this study compression pressure, balloon volume, and the duration of balloon compression were all varied. The mean compression time in this study was $1.15 \pm 0.21$ seconds and the mean pressure was $1520 \pm 101 \mathrm{~mm} \mathrm{Hg}$. At these values, the patients as a group had a lower rate of side effects and an equal incidence of pain relief, as compared with other series reported in the literature. The recurrence rate is comparable with previous studies, although longer follow-up data are not yet available.

Clinical results in numerous series of patients with $\mathrm{TN}$ treated by balloon compression have been published over the last 20 years, and other infrequent complications have been recognized. ${ }^{1,2,5,10,11,16,18-20,23,26,28,29}$ One case report on balloon compression described a death related to use of a sharp obturator that was passed beyond the foramen ovale into the cavernous sinus. ${ }^{27} \mathrm{~A}$ carotid cavernous fistula and an external carotid fistula have also been reported..$^{24}$ These incidents indicate that a cannula with a blunt obturator should be used to reach the foramen ovale through the cheek.

\section{CONCLUSIONS}

Percutaneous trigeminal compression continues to be an effective treatment for TN. In this study we have shown that trigeminal compression for 1 to 1.5 minutes with an intraluminal balloon pressure of 1140 to $1215 \mathrm{~mm} \mathrm{Hg}$ reduces the occurrence of masseter muscle weakness, dysesthesias, and severe numbness without reducing the degree of pain relief achieved in treating patients with classic TN.

\section{Acknowledgment}

We acknowledge the assistance of Brookes C. Brown in the preparation of this manuscript. 


\section{References}

1. Abdennebi B, Bouatta F, Chitti M, et al: Percutaneous balloon compression of the Gasserian ganglion in trigeminal neuralgia. Long-term results in 150 cases. Acta Neurochir 136:72-74, 1995

2. Belber CJ, Rak RA: Balloon compression rhizolysis in the surgical management of trigeminal neuralgia. Neurosurgery 20: 908-913, 1987

3. Brown JA: Percutaneous trigeminal nerve compression, in Batjer HH, Loftus CM (eds): Textbook of Neurological Surgery: Principles and Practice. Philadelphia: Lippincott Williams \& Wilkins, 2002, pp 2980-2985

4. Brown JA, Chittum CJ, Sabol D, et al: Percutaneous balloon compression of the trigeminal nerve for treatment of trigeminal neuralgia. Neurosurg Focus 1(2):E4, 1996

5. Brown JA, Gouda JJ: Percutaneous balloon compression of the trigeminal nerve. Neurosurg Clin N Am 8:53-62, 1997

6. Brown JA, Hoeflinger B, Long PB, et al: Axon and ganglion cell injury in rabbits after percutaneous trigeminal balloon compression. Neurosurgery 38:993-1003, 1996

7. Brown JA, McDaniel MD, Weaver MT: Percutaneous trigeminal nerve compression for treatment of trigeminal neuralgia: results in 50 patients. Neurosurgery 32:570-573, 1993

8. Brown JA, Preul MC: Percutaneous microcompression of the trigeminal ganglion. Neurosurgery 23:270, 1988

9. Brown JA, Preul MC: Percutaneous trigeminal ganglion compression for trigeminal neuralgia. Experience in 22 patients and review of the literature. J Neurosurg 70:900-904, 1989

10. Correa CF, Teixeira MJ: Balloon compression of the Gasserian ganglion for the treatment of trigeminal neuralgia. Stereotact Funct Neurosurg 71:83-89, 1998

11. Fiume D, Scarda G, Natali G: [Percutaneous microcompression of the gasserian ganglion. New treatment for trigeminal neuralgia.] Riv Neurol 55:387-391, 1985 (Ital)

12. Fraioli B, Esposito V, Guidetti B, et al: Treatment of trigeminal neuralgia by thermocoagulation, glycerolization, and percutaneous compression of the gasserian ganglion and/or retrogasserian rootlets: long-term results and therapeutic protocol. Neurosurgery 24:239-245, 1989

13. Lee ST, Chen JF: Percutaneous trigeminal ganglion balloon compression for treatment of trigeminal neuralgia - part I: pressure recordings. Surg Neurol 59:63-67, 2003

14. Lee ST, Chen JF: Percutaneous trigeminal ganglion balloon compression for treatment of trigeminal neuralgia, part II: results related to compression duration. Surg Neurol 60:149-154, 2003

15. Lichtor T, Mullan JF: A 10-year follow-up review of percutaneous microcompression of the trigeminal ganglion. J Neurosurg 72:49-54, 1990

16. Lobato RD, Rivas JJ, Sarabia R, et al: Percutaneous microcom- pression of the gasserian ganglion for trigeminal neuralgia. $\mathbf{J}$ Neurosurg 72:546-553, 1990

17. Lopez BC, Hamlyn PJ, Zakrzewska JM: Systematic review of ablative neurosurgical techniques for the treatment of trigeminal neuralgia. Neurosurgery 54:973-983, 2004

18. Meglio M, Cioni B: Percutaneous procedures for trigeminal neuralgia: microcompression versus radiofrequency thermocoagulation. Personal experience. Pain 38:9-16, 1989

19. Meglio M, Cioni B, d'Annunzio V: Percutaneous microcompression of the gasserian ganglion: personal experience. Acta Neurochir Suppl 39:142-143, 1987

20. Mizuno M, Saito K, Takayasu M, et al: Percutaneous microcompression of the trigeminal ganglion for elderly patients with trigeminal neuralgia and patients with atypical trigeminal neuralgia. Neurol Med Chir 40:347-351, 2000

21. Mullan S, Brown JA: Trigeminal neuralgia. Neurosurg Quart 6:267-288, 1996

22. Mullan S, Lichtor T: Percutaneous microcompression of the trigeminal ganglion for trigeminal neuralgia. J Neurosurg 59: 1007-1012, 1983

23. Natarajan M: Percutaneous trigeminal ganglion balloon compression: experience in 40 patients. Neurol India 48:330-332, 2000

24. Revuelta R, Nathal E, Balderrama J, et al: External carotid artery fistula due to microcompression of the gasserian ganglion for relief of trigeminal neuralgia. Case report. J Neurosurg 78:499-500, 1993

25. Shelden CH, Pudenz RH, Freshwater DB, et al: Compression rather than decompression for trigeminal neuralgia. J Neurosurg 12:123-126, 1955

26. Skirving DJ, Dan NG: A 20-year review of percutaneous balloon compression of the trigeminal ganglion. J Neurosurg 94: 913-917, 2001

27. Spaziante R, Cappabianca P, Peca C, et al: Subarachnoid hemorrhage and "normal pressure hydrocephalus": fatal complication of percutaneous microcompression of the gasserian ganglion. Case report. Neurosurgery 22:148-151, 1988

28. Urculo E, Arrazola M, Gereka L, et al: [Evaluation of the Mullan's technique in the treatment of trigeminal neuralgia.] Rev Neurol 27:477-484, 1998 (Spa)

29. Zanusso M, Curri D, Landi A, et al: Pressure monitoring inside Meckel's cave during percutaneous microcompression of gasserian ganglion. Stereotact Funct Neurosurg 56:37-43, 1991

Manuscript received March 15, 2005.

Accepted in final form April 8, 2005.

Address reprint requests to: Jeffrey A. Brown, M.D., Neurological Surgery, P.C., 600 Northern Boulevard \#118, Great Neck, New York 11021. email: jbrown@med.wayne.edu. 